\title{
EQUIVARIANT COMPLETE INVARIANCE PROPERTY ON A SUBSPACE OF TWO-INTERVAL WAVELETS
}

\author{
POOJA SINGH ${ }^{\dagger}$ AND DANIA MASOOD
}

\author{
Date of Receiving : 31.05 .2019 \\ Date of Revision : $\quad 11.07 .2019$ \\ Date of Acceptance : 28.07 .2019
}

\begin{abstract}
By providing a free action of the additive topological group of reals on a subspace $\omega$ of the space of all two-interval wavelets, we show that $\omega$ enjoys the complete invariance property. Further, considering $\omega$ as a topological transformation group with an action of the unit circle group, we obtain that $\omega$ possesses the equivariant complete invariance property.
\end{abstract}

\section{Introduction}

The notion of a flow has been axiomatized for providing precision to concepts available in dynamical systems. Its effect could be noticed in classifying topological spaces possessing properties such as the complete invariance property (CIP) and also the complete invariance property with respect to a homeomorphism $(\mathrm{CIPH})([6],[7],[8])$.

Although the notion of a wavelet is a recent development, its study rapidly grew twofold. Based on the core topics in Mathematics such as algebra, analysis and topology, wavelets while growing theoretically supported by the distribution theory and harmonic analysis, did not leave users of Mathematics to remain out of its reach. Evolving wavelets through tools in analysis gave way to workers interested in pure mathematics to investigate wavelets and many subspaces of wavelets from the point of view of topology. Subspaces of the wavelet space which have been taken into account include those of MRA wavelets (wavelets that arise from a multiresolution analysis) and MSF (minimally supported frequency) wavelets. To each one-dimensional MSF wavelet there is associated a specific subset of $\mathbb{R}$ possessing Lebesgue measure one. Precisely, this is the support of Fourier transform of the MSF wavelet, called a wavelet set. An MSF wavelet for which the wavelet set possesses $n$-components is called an $n$-interval wavelet. Such wavelets have been completely characterized for $n=2$ and symmetric $2 n$ intervals ([5], [1]).

2010 Mathematics Subject Classification. 42C40.

Key words and phrases. Wavelets, two-interval wavelet set, complete invariance property, equivariant complete invariance property.

Communicated by: I. Iglewska-Nowak

${ }^{\dagger}$ Corresponding author. 